\title{
Arteritis Mimicking Aortic Coarctation and Review of the Literature
}

\author{
Ning Bao ${ }^{\mathrm{a}}$, Hui-Ru Cao ${ }^{\mathrm{b}}$, Wen-Hui Zhang ${ }^{\mathrm{b}}$, Yu Zhang ${ }^{\mathrm{b}, \mathrm{c}}$
}

\begin{abstract}
Arteritis is a chronic non-specific inflammation involving the aorta and its main branches, coronary and pulmonary arteries which lead to vascular stenosis or occlusion. Compared with coronary atherosclerosis, primary hypertension and other common cardiovascular disease, arteritis is relatively rare disease. The aortic isthmus arteritist is rarer. Because of a variety of clinical manifestations, there is a little difficulty in early diagnosis and treatment with little experience. An 18-year-old Chinese girl was sent to our hospital for blood pressure $180 / 70 \mathrm{mmHg}$ in order to confirm the etiology of hypertension. One and a half years ago, she was diagnosed as "bronchiectasis" for hemoptysis and treated by using embolization intervention. Two months ago, she was again diagnosed as iron deficiency anaemia for fatigue. Physical examination discovered that right arm blood pressure was $180 / 70 \mathrm{mmHg}$ and left arm blood pressure $165 / 60 \mathrm{mmHg}$, but double lower limb blood pressure was not measured. Murmur was heard easy in bilateral carotid, subclavian, the back and heart valve auscultation area. However, ultrasound showed normal vascellum in bilateral carotid, subclavian other than decrease of blood flow velocity in double lower limb arteries. The aorta computed tomography angiography scanning found that there was only about $0.45 \mathrm{~cm}$ wide in the aortic isthmus with thicker wall. The results of hemoglobin, globulin, C-reaction protein and erythrocyte sedimentation rate were abnormal. The aortic isthmus arteritist and secondary hypertension were clearly diagnosed and treated by using anti-inflammatory, corticosteroids, immunosuppressive agents and antihypertensive drugs until erythrocyte sedimentation rate returned to normal. Finally, artificial vascular was replaced successfully by surgery. Now the patient fells very fine and
\end{abstract}

\footnotetext{
Manuscript accepted for publication March 19, 2013

${ }^{a}$ Medical undergraduate, Jilin University, Changchun 130021, Jilin Province, China

${ }^{\mathrm{b}}$ Department of Cardiology, the First Hospital of Jilin University, Changchun 130021, Jilin Province, China

${ }^{\mathrm{c} C}$ Corresponding author: Yu Zhang, Department of Cardiology, the First Hospital of Jilin University, Changchun 130021, Jilin Province, China. Email: zyu99@jlu.edu.cn
}

doi: http://dx.doi.org/10.4021/jmc1251e has already been working for more than two year. This case gives us the inspiration: A detailed examination to patient is very important, which avoid missed diagnosis or misdiagnosis and missed the best opportunity for treatment. As a doctor, we must have solid basic skills and do not underestimate the role of stethoscope in any time.

Keywords: Arteritis; Hypertension; Aortic coarctation; Interrupted aortic arch; Artificial vascular replacement

\section{Introduction}

Arteritis is a chronic nonspecific vasculitis affecting aorta and its main branches, coronary and pulmonary arteries which lead to vascular stenosis or occlusion. It is also called as Takayasu's arteritis which was first described by Takayasu in 1908. The systemic inflammatory response and organ ischemia are the main clinical manifestations. Compared with coronary atherosclerosis, primary hypertension and other common cardiovascular disease, Takayasu's arteritis is relatively rare disease. Etiology and pathogensis is not clear yet. Because of a variety of clinical manifestations, there is a little difficulty in early diagnosis and treatment with little experience [1]. In this paper, we will report a case with Takayasu's arteritis mimicking aortic coarctation and its review of the literature.

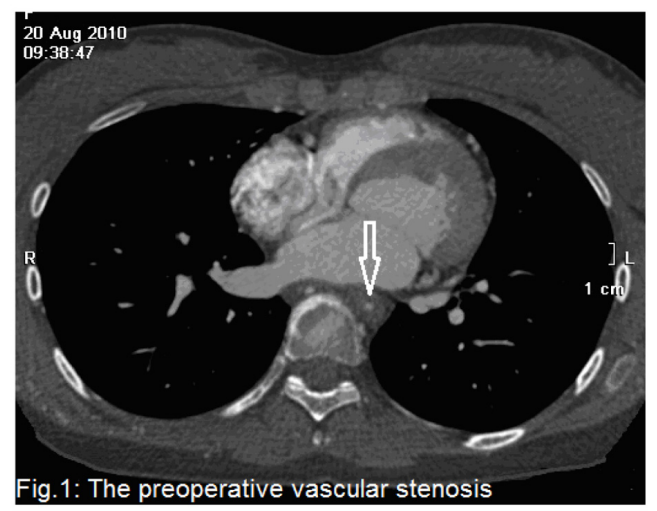

Figure 1. The preoperative vascular stenosis. 


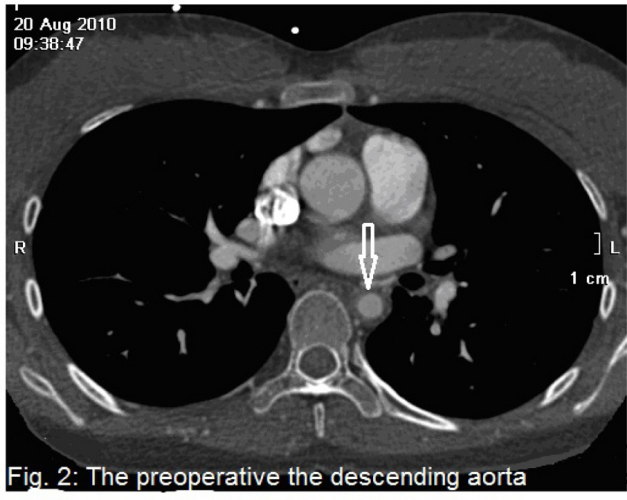

Figure 2. The preoperative the descending aorta.

\section{Case Report}

An 18-year-old Chinese girl, who was diagnosed as hypertension for headache, anxiety, frowsty, nausea, vomiting and blood pressure (BP) 180/70 $\mathrm{mmHg}$ in local hospital eight days ago, was sent to our hospital in 2009 July in order to confirm the etiology of hypertension. Past medical history: One and a half years ago, she was diagnosed as "bronchiectasis" for hemoptysis and treated by using embolization intervention. Two months ago, she was again diagnosed as iron deficiency anaemia for fatigue. Physical examination detected: Right arm BP was 180/70 $\mathrm{mmHg}$ and left arm BP 165/60 mmHg. Double lower limb BP was not measured. Vascular murmur was heard in bilateral carotid, subclavian and the back, however, not in abdominal. 4/6 pan systolic murmur and stronger heart sound were heard in each valve auscultation area. Bilateral radial pulses were symmetrical, but bilateral femoral, popliteal and dorsal artery of foot was weakened. Heart color Doppler showed increase of left ventricular diameter and left ventricular systolic function impairment. Vascular ultrasound showed decrease of blood

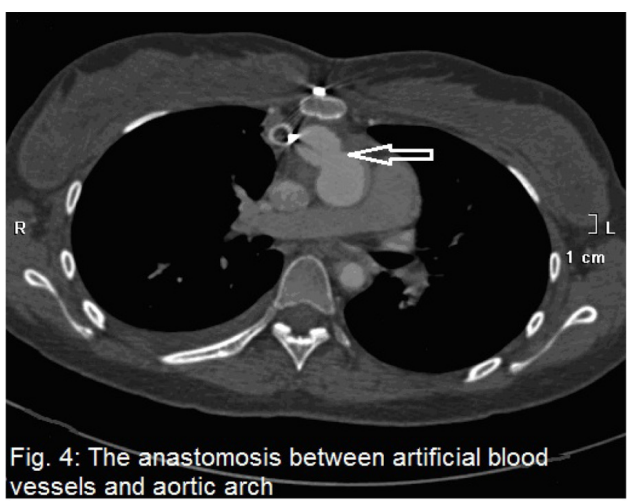

Figure 4. The anastomosis between artificial blood vessels and aortic arch.

flow velocity in double lower limb arteries, but normal in bilateral carotid, subclavian. Thicker wall and narrower lumen in the distal aortic arch and part of the descending aorta were demonstrated by the aorta computed tomography angiography scanning (CTA) scanning which the smallest lumen was about $0.45 \mathrm{~cm}$ and with collateral circulation (Fig. 1-3) (preoperative). Results of laboratory tests were hemoglobin (HB) $9.5 \mathrm{~g} / \mathrm{L}$, globulin $38.4 \mathrm{mmol} / \mathrm{L}, \mathrm{C}$-reaction protein (CRP) $29 \mathrm{mg} / \mathrm{L}$ and erythrocyte sedimentation rate (ESR) 55 $\mathrm{mm} / \mathrm{h}$.

According to above, the patient was diagnosed as Takayasu arteritis and secondary hypertension and treated by using anti-inflammatory, corticosteroids, immunosuppressive agents and antihypertensive drugs until ESR returned to normal. Finally, artificial vascular was successfully anastomsed by surgery from aortic arch to descending aorta in 2010 September (Fig. 4-6) (postoperative). After operation, her blood pressure returned to normal. She has already been working for more than two years without any symptoms. The left ventricular diameter and systolic function have returned to normal by echocardiography review.

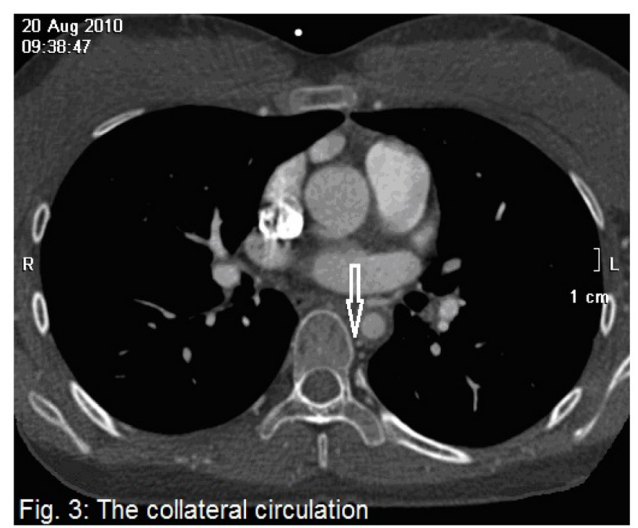

Figure 3. The collateral circulation.

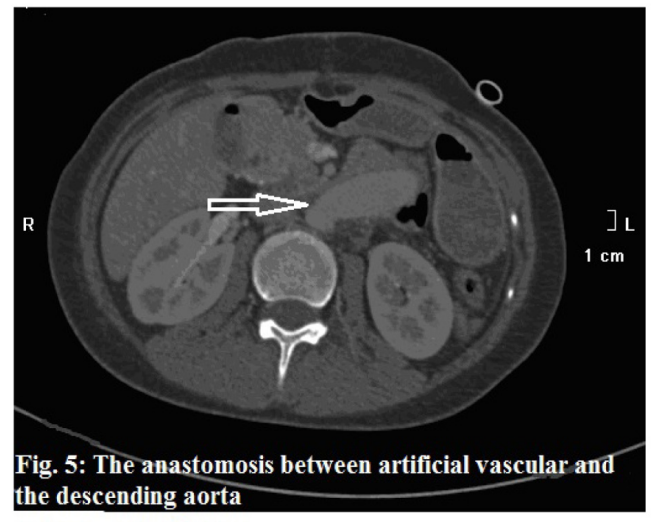

Figure 5. The anastomosis between artificial vascular and the descending aorta. 


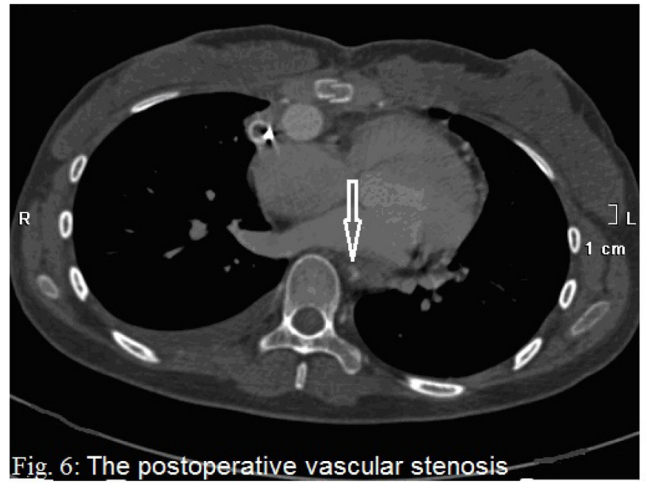

Figure 6. The postoperative vascular stenosis.

\section{Discussion}

The diagnostic criteria of Takayasu's arteritis formulated in 1990 by the American rheumatism association: 1). Onset age less than forty years old; 2). Body intermittent claudication; 3). Unilateral or bilateral brachial artery beat abate; 4). More than $10 \mathrm{mmHg}$ SBP difference in double upper limb; 5). Vascular murmur; 6). Arterial abnormality revealed by digital subtraction angiography (DSA) or CTA. Takayasu's arteritis can be made in accord with three above [2, 3]. Diagnosis of this patient was unquestionable. Sunamori et al discovered through autopsy that all of the patients with Takayasu's arteritis were accompanied by different degree change of pulmonary. Haemoptysis might be considered as the earliest symptoms of Takayasu's arteritis [4]. So, in order to diagnose early we should make examination of CTA to the patient with haemoptysis, especially young women. Aortic arch and its branch artery or abdominal aorta and renal artery were often involved in $84 \%$ patients with arteritis [2]. But aortic isthmus involvement is very rare.

We should pay more attention to identify with the congenital coarctation of aorta, which was considered as follows: 1). More incidence in men than women 3 - 5:1. 2). Thoracic-descending aorta more involved, the aorta spondylolysis in infantile and artery catheter junction in adult. 3). Specific and limited stenosis found by angiography. 4). Without systemic disease activity and abdominal murmurs [5]. However, characteristics in this patient were as follows: 1). Increase of ESR and CRP indicating the infective diseases as well as its activity. 2). Vascular murmur representing stenosis of vascular lumen. 3). Thickening arterial wall also clew Takayasu's arteritis. 4). One of Takayasu's arteritis etiology was known as estrogen disorder in which lead to abnormal menstruation and hematopoietic inhibition. Onethird of patients with Takayasu's arteritis are accompanied by anemia [6]. 5). Shortage of abdominal and lower limb blood supply caused reflexively the high BP which lead to left ventricular enlargement and cardiac dysfunction finally. Therefore, the patient was diagnosed as Takayasu's arteritis, instead of the congenital coarctation of the aorta. Similarly, interrupted aortic arch in the adult was also differentiated, which is a disruption of aorta continuity distal to the left subclavian artery and a rare congenital vascular malformation associated with a high mortality rate in infancy $[7,8]$.

Although the pathogenesis of Takayasu's arteritis remains unclear, the interaction of many factors, such as autoimmunity, inflammation, genetic and environmental factors and so on, is involved in the occurrence and development of Takayasu's arteritis [3]. Interventional therapy may face the risk of a ruptured vessel. Thus, surgical operation is the best choice to this kind of focal Takayasu's arteritis. Before operation anti-inflammatory, corticosteroids, immunosuppressive agents and antihypertensive drugs must be used until ESR returned to normal in order to reduce the operation risk.

This case gives us inspiration: A detailed examination to patient is very important, which can avoid missed diagnosis or misdiagnosis or missed the best opportunity for treatment. If the patient had been diagnosed correctly in one and a half years ago or two months ago, she would not have suffered later body pain. If this had still not been diagnosed as Takayasu's arteritis, the patient could have beard the onset of complications caused by poor control of hypertension, which leads to disability or death [9]. This would be a very bad result to patient and her family. So, as a doctor, we must have solid basic skills and do not underestimate the role of stethoscope in any time.

\section{Grant Support and Other Assistance}

None.

\section{Conflicts of Interest}

The authors declare that they have no competing interests.

\section{Authors' Contributions}

All authors involved in the management of patient. $\mathrm{BN}$ and $\mathrm{ZY}$ wrote and reviewed the manuscript. All authors read and approved the final manuscript.

\section{References}

1. Feng TJ, Wang ZW, Zhang Y. Diagnosis and treatment of Takayasu arteritis. Chinese Journal of hypertension. 2008; 16(6): 569-572.

2. Ye RG, Lu ZY, Xie Y and Wang C. The sixth edition of internal. Beijing: People's medical publishing house, 2004: 915-917. 
3. Wen D, Du X, Ma CS. Takayasu arteritis: diagnosis, treatment and prognosis. Int Rev Immunol. 2012;31(6):462473.

4. Sunamori M, Hatano R, Yamada T, Tsukuura T, Sakamoto T. Aortitis syndrome due to Takayasu's disease. A guideline for the surgical indication. J Cardiovasc Surg (Torino). 1976;17(5):443-456.

5. Wei M. Diagnosis and differential diagnosis of cardiovascular diseases. Tianjin: Tianjin science and technology publishing house, 2004: 270-274.

6. Jiang JJ. Peripheral vascular disease. Beijing: Science and technology literature press, 2001: 63-76.

7. Bao N, Zhang Y, Zhang ZU, Cao HR, Zhang WH. Interrupted aortic arch in a 42-year-old man. J South Med Univ. 2012; 32 (4): 597-598.

8. Bryl M, Guzinski M, Rabczynski M, Waliszewska-Prosol M, Garcarek J, Adamiec R, Sasiadek M. Imaging difficulties in Takayasu arteritis - case report and review of the literature. Pol J Radiol. 2012;77(4):67-71.

9. Silver M. Takayasu's Arteritis - An Unusual Cause of Stroke in a Young Patient. West J Emerg Med. 2012;13(6):484-487. 